\title{
Identification of B-cell translocation gene 1-controlled gene networks in diffuse large B-cell lymphoma: A study based on bioinformatics analysis
}

\author{
WEI YAN ${ }^{1}$, SHAWN XIANG LI ${ }^{2}$, HONGYU GAO $^{1}$ and WEI YANG ${ }^{1}$ \\ ${ }^{1}$ Department of Hematology, Shengjing Hospital of China Medical University; \\ ${ }^{2}$ International College, China Medical University, Shenyang, Liaoning 110022, P.R. China
}

Received March 15, 2018; Accepted November 16, 2018

DOI: $10.3892 / 01.2019 .9900$

\begin{abstract}
B-cell translocation gene 1 (BTG1) is a member of the BTG/transducer of Erb family. The present study evaluated the impact of BTG1 gene expression on the clinical outcome of diffuse large B-cell lymphoma (DLBCL) and investigated potential mechanisms using the Gene Expression Omnibus (GEO) database. The gene expression profile datasets GSE31312, GSE10846, GSE65420 and GSE87371 were downloaded from the GEO database. BTG1 expression and clinicopathological data were obtained from the GSE31312 dataset. In 498 cases, the expression of BTG1 in DLBCL was associated with treatment response $\left(\chi^{2}=19.020 ; \mathrm{P}<0.001\right)$ and International Prognostic Index score $\left(\chi^{2}=5.320 ; \mathrm{P}=0.025\right)$. Using the Kaplan-Meier method, it was identified that the expression of BTG1 was associated with overall survival (OS) and progression-free survival (PFS) times. Univariate and multivariate Cox regression analysis demonstrated that BTG1 was an independent predictive factor for OS and PFS. From the overlapping analysis of 407 BTG1-associated genes and
\end{abstract}

Correspondence to: Professor Wei Yang, Department of Hematology, Shengjing Hospital of China Medical University, 39 Huaxiang Road, Shenyang, Liaoning 110022, P.R. China

E-mail: cmu-yangwei@hotmail.com

Abbreviations: BTG1, B-cell translocation gene 1; DLBCL, diffuse large B-cell lymphoma; GEO, Gene Expression Omnibus; STRING, Search Tool for the Retrieval of Interacting Genes/Proteins; OS, overall survival; PFS, progression-free survival; IPI, International Prognostic Index; GCB, germinal center B-like; BCR, B-cell receptor; HR, hazard ratio; GO, Gene Ontology; KEGG, Kyoto Encyclopedia of Genes and Genomes; PPI, protein-protein interaction; MCODE, Molecular Complex Detection; CR, complete response; $\mathrm{PR}$, partial response; $\mathrm{PD}$, progressive disease; $\mathrm{SD}$, stable disease; DEG, differentially-expressed gene; RP, ribosomal protein; mTOR, mammalian target of rapamycin

Key words: diffuse large B-cell lymphoma, B-cell translocation gene 1-associated genes, prognosis, enrichment analysis, interaction network
22,187 DLBCL-associated genes, 401 genes were identified as BTG1-associated DLBCL genes. Pathway analysis revealed that BTG1-associated DLBCL genes were associated with cancer progression and DLBCL signaling pathways. Subsequently, a protein-protein interaction network was constructed of the BTG1-associated genes, which consisted of 235 genes and 601 interactions. Additionally, 24 genes with high degrees in the network were identified as hub genes, which included genes associated with 'ribosome' [ribosomal protein (RP) L11, RPL3, RPS29, RPL19, RPL15 and RPL12], 'cell cycle' (ubiquitin carboxyl extension protein 52, ATM and Ras homolog family member $\mathrm{H}$ ), 'mitogen-activated protein kinase pathway' (mitogen-activated protein kinase 1), 'histone modification' (ASH1-like protein) and 'transcription/translation' (eukaryotic translation initiation factor 3 subunit E, eukaryotic translation elongation factor $1 \delta$, transcription termination factor 1 , cAMP responsive element binding protein 1 and RNA polymerase II subunit F). In conclusion, BTG1 may serve as a predictive biomarker for DLBCL prognosis. Additionally, bioinformatics analysis indicated that BTG1 may exhibit key functions in the progression and development of DLBCL.

\section{Introduction}

Despite improvements in diagnostic techniques, the incidence rate of lymphoma has been increased by $75 \%$ in the past 20 years (1). Less than half of the patients who are diagnosed with diffuse large B-cell lymphoma (DLBCL) achieve complete remission (2). Patients with DLBCL exhibit various clinical outcomes due to tumors possessing different histology, morphology and clinical features (3). Treatment for patients with DLBCL includes combinations of radiation therapy, chemotherapy and targeted therapy. The long-term remission rate of the disease has improved with the introduction of rituximab; however, this treatment has poor efficacy in certain patients (4). Therefore, there is an increasing requirement to further understand the molecular mechanisms underlying the disease. This would assist with survival prediction and enable the design of improved targeted therapeutic strategies.

DLBCL is one of the most studied diseases for prognostic markers. Since its publication, the International Prognostic Index (IPI) has been used to predict the prognosis of patients 
with DLBCL. Immunohistochemistry has been used to classify DLBCL into germinal center B-like (GCB) and non-GCB subgroups, with various positive staining combinations of cluster of differentiation 10 , mutated melanoma-associated antigen 1,B-cell lymphoma 6 and CD138 (5). Numerous studies have confirmed that GCB subgroups improve prognosis estimations of DLBCL (6-8). According to the 2016 World Health Organization Classification of Tumors of Haematopoietic and Lymphoid Tissue, Epstein-Barr virus-positive DLBCL is frequently diagnosed in immunocompromised patients and demonstrates a poor response to treatment (9). Previously, a number of studies investigated MYC. Multivariate analysis illustrated that extra copies of MYC and MYC rearrangement in DLBCL are independent poor prognostic factors (10). Numerous studies confirmed that the development and progression of DLBCL is associated with multiple signaling pathways, including the Wnt, nuclear factor- $\kappa \mathrm{B}(\mathrm{NF}-\kappa \mathrm{B})$, mammalian target of rapamycin (mTOR) and B-cell receptor (BCR) signaling pathways $(11,12)$.

B-cell translocation gene 1 (BTG1) is a member of the BTG/transducer of Erb (TOB) family. This family consists of six members, BTG1, BTG2/PC3/TIS21, BTG3, BTG4/PC3B, TOB1 and TOB2, which regulate cell cycle progression and differentiation, and inhibit proliferation (13). The BTG/TOB family consists of two characteristic and conserved domains, Box A and Box B (14). Additionally, BTG/TOB proteins are nuclear proteins that are transported into the nucleus by nuclear localization signaling (15). Human BTG1 is located on chromosome 12q22 and consists of 4,704 nucleotides that encode 171 amino acids and a $19 \mathrm{kDa}$ protein (16). BTG1 promotes apoptosis, stimulates cellular differentiation, maintains cell cycle progression and inhibits proliferation, and therefore functions as a tumor suppressor gene (17). A previous study identified that BTG1 expression is increased in the G0/G1 phase and decreased in the G1 phase of the cell cycle (18). Therefore, BTG1 is considered to be a potential suppressor gene due to its effects on cell cycle progression and proliferation (19). BTG1 interacts with arginine $\mathrm{N}$-methyltransferase 1 in vitro, which regulates transcription and affects cytokine signaling pathways (20). BTG1 enhances the inhibitory function of homeobox B9-mediated transcription (21). Additionally, overexpression of BTG1 enhances apoptosis of NIH/3T3 cells (22). A recent study revealed that BTG1 serves as a tumor suppressor in B-cell precursor acute lymphoblastic leukemia (23). Similarly, another study demonstrated that BTG1 acts as a regulator of B-cell differentiation, which supports a role of BTG1 as a tumor suppressor in B-cell malignancies (24). However, a limited number of studies have performed global network analysis for BTG1, which limits the investigation of BTGl's role in DLBCL.

The present study investigated the association between BTG1 expression and clinicopathological parameters in patients with DLBCL. Subsequently, the prognostic value and functional mechanism of BTG1 in DLBCL were further analyzed by utilizing certain bioinformatics methods. Additionally, Oncomine analysis was performed, which revealed that BTG1 was downregulated in DLBCL. Furthermore, the Search Tool for the Retrieval of Interacting Genes/Proteins (STRING) database and Cytoscape analysis demonstrated that hub genes of BTG1-associated DLBCL interaction networks were enriched in 'Ribosome', 'Cell cycle' and 'B cell receptor signaling pathway'. In conclusion, BTG1 may serve as an independent predictor for DLBCL prognosis and as a potential therapeutic target.

\section{Materials and methods}

Patient characteristics from the Gene Expression Omnibus (GEO) database and statistical analysis. A gene expression profile, GSE31312 (25), was downloaded from the GEO database (http://www.ncbi.nlm.nih.gov/geo). GSE31312 is a human DLBCL expression profile that contains BTG1 expression data, which were sequenced using the GPL570 platform (Affymetrix Human Genome U133 Plus 2.0 Array). GSE31312 contains 498 samples of DLBCL, of which 470 samples have clinical data. According to GSE31312 data, the median value of BTG1 expression was calculated and the 470 patients with BTG1 expression $\geq 4.34$ were placed in the high expression group and patients with BTG1 expression $<4.34$ were placed in the low expression group. The association between BTG1 expression level and numerous factors, including sex, age, Ann Arbor stage (26), Eastern Cooperative Oncology Group (ECOG) score, subtype, IPI score, B symptoms, bulky disease, lactate dehydrogenase (LDH) level, treatment response and survival data, were analyzed by extraction of clinical data from GSE31312. All analysis was performed using SPSS 19.0 software (IBM Corp., Armonk, NY, USA) and GraphPad Prism 5.0 software (GraphPad Software, Inc., La Jolla, CA, USA). Associations between BTG1 expression and clinical parameters were examined with the $\chi^{2}$ test. Survival analysis was performed using the Kaplan-Meier method and differences were analyzed by log-rank test. Univariate and multivariate Cox proportional hazards regression analyses were performed to identify independent predictors. The hazard ratios (HRs) and 95\% confidence interval (CIs) of the prognostic factors were calculated. $\mathrm{P}<0.05$ was considered to indicate a statistically significant difference.

Microarray data and data processing. Expression levels of BTG1 in DLBCL were obtained from the Oncomine database (http://www.oncomine.com/resource/main.html). The GSE31312 (25), GSE10846 (27) and GSE87371 (28) datasets were downloaded from the GEO database and the R2 platform (https://hgserver1.amc.nl/cgi-bin/r2/main.cgi) was applied to identify the BTG1-associated genes. The cut-off point was defined as: $\mathrm{P}<0.01$ and PresCalls $\geq 1$. Only BTG1-associated genes identified in all three independent datasets were selected. Furthermore, GEO2R (http://www.ncbi.nlm.nih. gov/geo/geo2r/) was applied to reveal differentially-expressed genes (DEGs) in DLBCL, compared with normal lymphocytes. The following cut-off criteria was applied: $\mathrm{P}<0.05$ and $\mid \log$ (fold-change) $\mid>1$. A Venn diagram was generated to visualize the overlapping BTG1-associated genes and DLBCL-associated DEGs. The resulting overlapping genes were defined as BTG1-associated DLBCL genes.

Gene Ontology (GO) and Kyoto Encyclopedia of Genes and Genomes (KEGG) pathway analysis. GO and pathway analysis were performed using the Database for Annotation, 
Table I. Association between BTG1 expression level and clinical characteristics obtained from the GSE31312 dataset.

\begin{tabular}{|c|c|c|c|c|c|}
\hline Characteristic & Case, n (\%) & Low BTG1 expression, $\mathrm{n}$ & High BTG1 expression, $\mathrm{n}$ & $\chi^{2}$ value & P-value \\
\hline Sex & & & & 0.218 & 0.709 \\
\hline Male & $199(42.3)$ & 138 & 133 & & \\
\hline Female & $271(57.7)$ & 97 & 102 & & \\
\hline Age, years & & & & 0.690 & 0.460 \\
\hline$<63$ & $229(48.7)$ & 110 & 119 & & \\
\hline$\geq 63$ & $241(51.3)$ & 125 & 116 & & \\
\hline Stage & & & & 0.000 & 1.000 \\
\hline $\mathrm{I} / \mathrm{II}$ & $220(46.8)$ & 110 & 110 & & \\
\hline III/IV & $250(53.2)$ & 125 & 125 & & \\
\hline ECOG score & & & & 0.209 & 0.732 \\
\hline Low & $374(79.6)$ & 185 & 189 & & \\
\hline High & $96(20.4)$ & 50 & 46 & & \\
\hline Subtype & & & & 0.034 & 0.926 \\
\hline Non-GCB & $222(47.2)$ & 112 & 110 & & \\
\hline GCB & $248(52.8)$ & 123 & 125 & & \\
\hline IPI score & & & & 5.320 & 0.025 \\
\hline Low & $274(64.6)$ & 135 & 139 & & \\
\hline High & $150(35.4)$ & 79 & 71 & & \\
\hline B symptom & & & & 0.513 & 0.526 \\
\hline No & $276(67.6)$ & 138 & 138 & & \\
\hline Yes & $132(32.4)$ & 61 & 71 & & \\
\hline Bulky disease & & & & 0.141 & 0.724 \\
\hline No & $268(73.2)$ & 129 & 139 & & \\
\hline Yes & $98(26.8)$ & 45 & 53 & & \\
\hline LDH level & & & & 0.071 & 0.839 \\
\hline Normal & $148(34.7)$ & 76 & 72 & & \\
\hline High & $278(65.3)$ & 139 & 139 & & \\
\hline Treatment response & & & & 19.020 & $<0.001$ \\
\hline $\mathrm{CR}$ & $354(75.3)$ & 157 & 197 & & \\
\hline PR & $72(15.3)$ & 48 & 24 & & \\
\hline PD & $24(5.1)$ & 15 & 9 & & \\
\hline SD & $20(4.3)$ & 15 & 5 & & \\
\hline
\end{tabular}

BTG1, B-cell translocation gene 1; ECOG, Eastern Cooperative Oncology Group; GCB, germinal center B-like; IPI, International Prognostic Index; LDH, lactate dehydrogenase; CR, complete response; PR, partial response; PD, progressive disease; SD, stable disease.

Visualization and Integrated Discovery (http://david.abcc. ncifcrf.gov/), and the KEGG database (29). $\mathrm{P}<0.05$ indicated a statistically significant enriched GO and pathway term for the BTG1-associated DLBCL genes.

Establishment of a protein-protein interaction (PPI) network and cluster selection. The STRING database (http://string-db. org) was used to predict interaction networks of the protein products of BTG1-associated DLBCL genes. A confidence score of $\geq 0.4$ was set as the cut-off point. Cytoscape 3.5.1 software (Institute for Systems Biology, Seattle, WA, USA) was used to construct the PPI networks for BTG1-associated DLBCL genes. The hub genes were identified using the cytohubba plugin in Cytoscape software and a degree $\geq 17$ was set as the cut-off criterion. Molecular Complex Detection (MCODE) v1.5 (30) was subsequently used to reveal clusters of genes in the PPI network.

\section{Results}

Patient characteristics from the GSE31312 dataset. Patient data downloaded from GEO database are presented in Table I. The patients included 199 males and 271 females, and the median age at diagnosis was 63 years (range, 18-92 years). All patients were assessed according to the Ann Arbor staging system (26) and patients were divided into a low stage group (I and II; 220 patients) or high stage group (III and IV; 250 patients). A total of 374 patients had a low ECOG score $(\leq 1)$ and 96 had a 

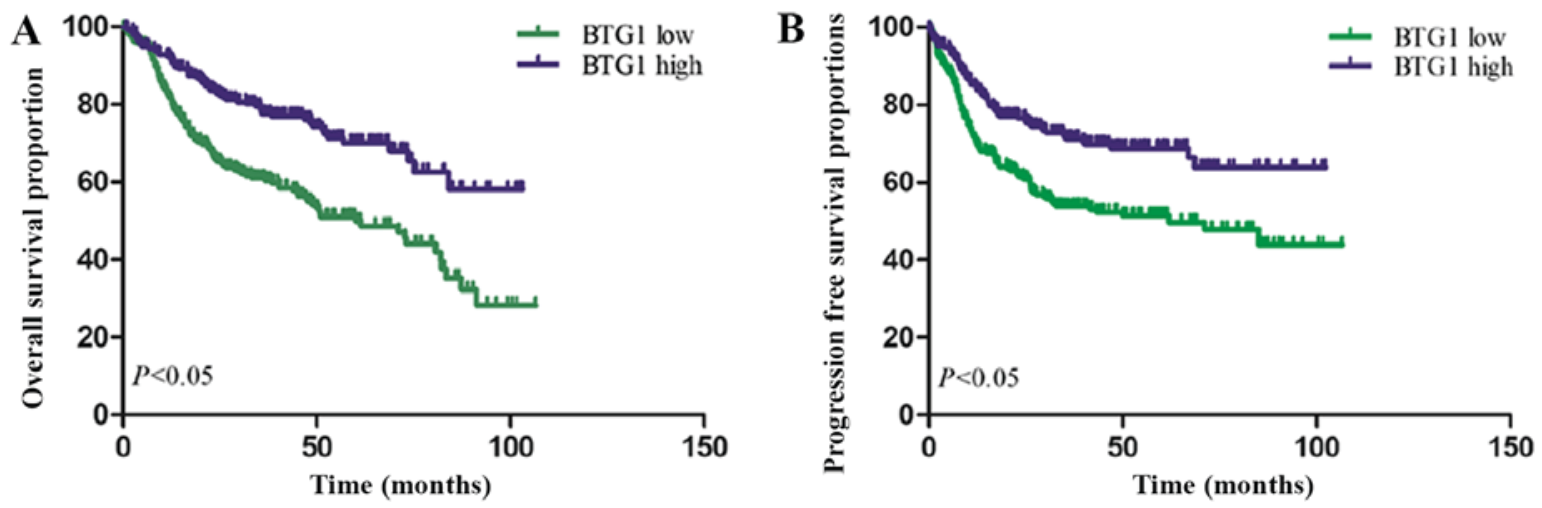

Figure 1. Kaplan-Meier survival curves for OS and PFS time of patients with diffuse large B-cell lymphoma stratified by median BTG1 expression level. (A) OS curve. (B) PFS curve. OS, overall survival; PFS, progression-free survival; BTG1, B-cell translocation gene 1.

A

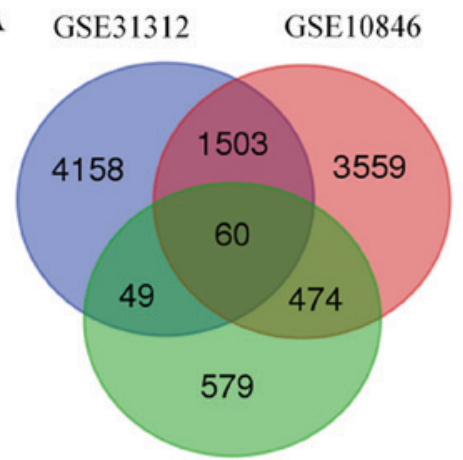

GSE87371
B

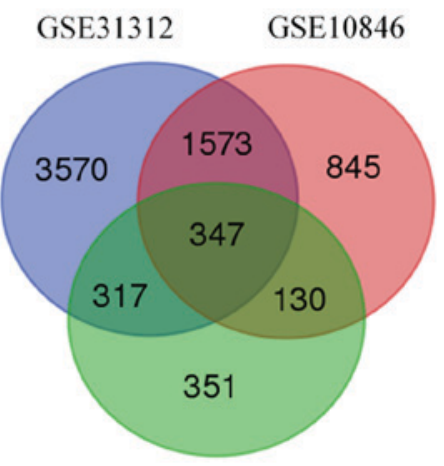

GSE87371

Figure 2. Identification of B-cell translocation gene 1-associated genes. (A) Downregulated and (B) upregulated genes in mRNA expression profiling datasets GSE31312, GSE10846 and GSE87371.

high ECOG score (>1). Information regarding IPI score was available for 424 cases. A total of 274 patients had a low IPI score ( $\leq 2)$ and 150 had a high IPI score ( $>3)$. Only 408 of the 470 cases had B symptom data, 366 cases had bulky disease data and 426 cases had LDH level data. The 470 cases were divided into complete response (CR), partial response (PR), progressive disease (PD) and stable disease (SD) groups, according to their treatment response.

Associations between BTG1 expression level and the clinical characteristics of patients with DLBCL. A total of 470 samples in the GSE31312 dataset contained BTG1 expression data. The associations between BTG1 expression level and clinical features of patients with DLBCL were investigated (Table I). It was identified that the BTG1 expression level was significantly different in treatment response $(\mathrm{P}<0.001)$ and IPI score $(\mathrm{P}=0.025)$ groups. However, no significant difference in BTG1 expression level was observed for age, sex, stage, subtype, ECOG score, B symptom, bulky disease or LDH level $(\mathrm{P}>0.05)$.

Prognostic performance of BTG1 for DLBCL. Based on the median expression level of BTG1, Kaplan-Meier analysis was preformed to estimate overall survival (OS_ and progression-free survival (PFS) times. As demonstrated in Fig. 1, Kaplan-Meier survival curves revealed that patients with low

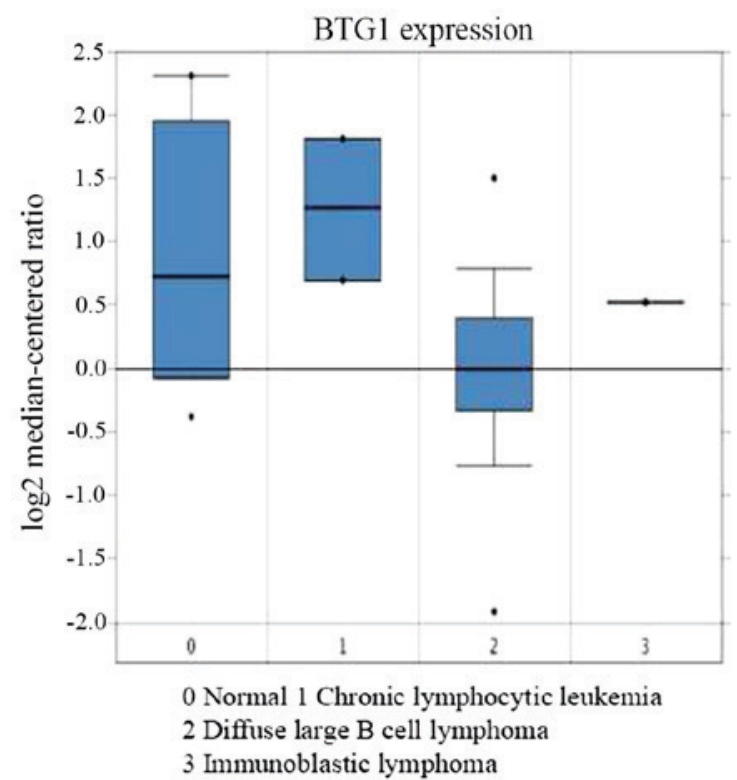

Figure 3. The mRNA expression level of BTG1 in diffuse large B-cell lymphoma, obtained from the Oncomine database. Data are present as the mean \pm standard deviation. BTG1, B-cell translocation gene 1 .

BTG1 expression exhibited a reduced OS time, compared with patients with high BTG1 expression $(\mathrm{P}<0.001)$. Furthermore, 
Table II. Univariate and multivariate Cox regression analysis for patients with diffuse large B-cell lymphoma.

A, Overall survival

\begin{tabular}{lcrrr}
\hline & \multicolumn{2}{c}{ Univariate analysis } & & Multivariate analysis \\
\cline { 2 - 3 } Variable & HR $(95 \%$ CI $)$ & P-value & HR (95\% CI) \\
\hline Sex (males vs. females) & $1.050(0.775-1.423)$ & 0.752 & \\
Age ( $\geq 63$ vs. $<63)$ years & $1.697(1.246-2.312)$ & 0.752 & $1.742(1.244-2.441)$ \\
Stage (low vs. high) & $2.307(1.668-3.189)$ & $<0.001$ & $1.594(1.103-2.302)$ \\
ECOG score (low vs. high) & $2.021(1.450-2.818)$ & $<0.001$ & $1.978(1.376-2.844)$ \\
Subtype (non-GCB vs. GCB) & $0.668(0.494-0.904)$ & 0.009 & $1.978(1.376-2.844)$ \\
IPI score (low vs. high) & $1.411(1.026-1.940)$ & 0.034 & 0.001 \\
B symptom (no vs. yes) & $1.105(1.787-1.551)$ & 0.565 & $<0.001$ \\
LDH (normal vs. high) & $1.120(0.803-1.563)$ & 0.503 & $<0.001$ \\
Bulky disease (no vs. yes) & $1.051(0.732-1.509)$ & 0.787 & \\
Treatment response (CR+PR vs. PD+SD) & $2.605(2.828-2.990)$ & $<0.001$ & $2.612(2.214-3.081)$ \\
BTG1 expression (low vs. high) & $2.066(1.508-2.829)$ & $<0.001$ & $1.692(1.193-2.401)$ \\
\hline
\end{tabular}

B, Progression free survival

\begin{tabular}{|c|c|c|c|c|}
\hline \multirow[b]{2}{*}{ Variable } & \multicolumn{2}{|c|}{ Univariate analysis } & \multicolumn{2}{|c|}{ Multivariate analysis } \\
\hline & HR $(95 \% \mathrm{CI})$ & P-value & HR $(95 \%$ CI $)$ & P-value \\
\hline Sex (males vs. females) & $0.864(0.639-1.169)$ & 0.343 & & \\
\hline Age $(\geq 63$ vs. $<63)$, years & $1.156(0.855-1.563)$ & 0.346 & & \\
\hline Stage (low vs. high) & $2.469(1.781-3.422)$ & $<0.001$ & $1.538(1.063-2.226)$ & 0.022 \\
\hline ECOG score (low vs. high) & $1.678(1.191-2.366)$ & 0.003 & $1.187(0.815-1.729)$ & 0.371 \\
\hline Subtype (non-GCB vs. GCB) & $0.624(0.461-0.846)$ & 0.002 & $0.563(0.406-0.782)$ & 0.001 \\
\hline IPI score (low vs. high) & $1.545(1.124-2.124)$ & 0.007 & & \\
\hline B symptom (no vs. yes) & $1.261(0.902-1.763)$ & 0.125 & & \\
\hline LDH (normal vs. high) & $1.078(0.774-1.501)$ & 0.659 & & \\
\hline Bulky disease (no vs. yes) & $1.175(0.820-1.682)$ & 0.379 & & \\
\hline Treatment response (CR+PR vs. PD+SD) & $2.401(2.095-2.751)$ & $<0.001$ & $2.220(1.889-2.607)$ & $<0.001$ \\
\hline BTG1 expression (low vs. high) & $1.801(1.324-2.449)$ & $<0.001$ & $1.403(1.004-1.960)$ & 0.047 \\
\hline
\end{tabular}

HR, hazard ratio; CI, confidence interval; BTG1, B-cell translocation gene 1; ECOG, Eastern Cooperative Oncology Group; GCB, germinal center B-like; IPI, International Prognostic Index; LDH, lactate dehydrogenase; CR, complete response; PR, partial response; PD, progressive disease; $\mathrm{SD}$, stable disease.

low BTG1 expression was identified to be associated with a reduced PFS time in patients with DLBCL $(\mathrm{P}<0.001)$.

To assess whether BTG1 is an independent prognostic factor for DLBCL, univariate and multivariate Cox regression analysis was performed. The results revealed that age (HR, 1.742; 95\% CI, 1.244-2.441; P=0.001), stage (HR, 1.594; 95\% CI, 1.103-2.302; $\mathrm{P}=0.013$ ), ECOG score (HR, 1.978; 95\% CI, 1.376-2.844; P<0.001), subtype (HR, 1.978; 95\% CI, 1.376-2.844; $\mathrm{P}<0.001)$, treatment response (HR, 2.612; 95\% CI, 2.214-3.081; $\mathrm{P}<0.001)$ and BTG1 expression (HR, 1.692; 95\% CI, 1.193-2.401; $\mathrm{P}=0.003$ ) were independent prognostic factors for OS time. Subsequently, multivariate Cox regression analysis was performed to determine the independence of the prognostic power of BTG1 for PFS time. The results demonstrated that stage (HR, 1.538; 95\% CI, 1.063-2.226; $\mathrm{P}=0.022)$, subtype (HR, 0.563; 95\% CI, 0.406-0.782; $\mathrm{P}=0.001)$, treatment response (HR, 2.220; 95\% CI, 1.889-2.607; $\mathrm{P}<0.001)$ and BTG1 expression (HR, 1.403; 95\% CI, 1.004-1.960; $\mathrm{P}=0.047$ ) could predict a reduced PFS time for patients with DLBCL (Table II).

Analysis of BTG1-associated genes. BTG1-associated genes from DLBCL gene expression profiling datasets were identified using the R2 platform and the following criteria: $\mathrm{P}<0.01$ and PresCalls $\geq 1$. A total of 11,577, 8,491 and 2,307 genes were identified to be associated with BTG1 in the GSE1312, GSE10846 and GSE87371 datasets, respectively. Additionally, 407 BTG1-associated genes were identified in all three 
A DEGs of DLBCL

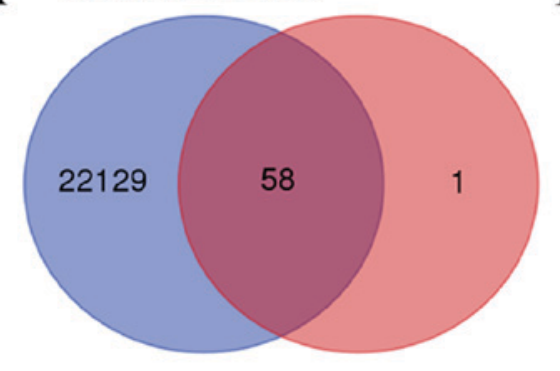

BTG1-related genes
B DEGs of DLBCL

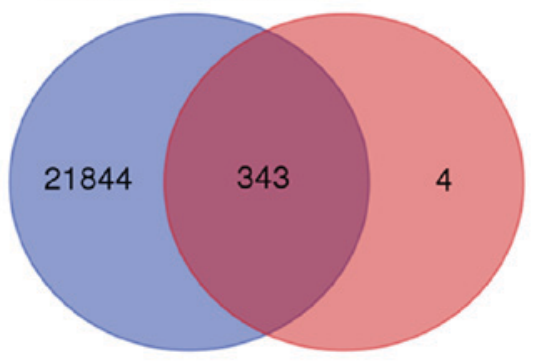

BTG1-related genes

Figure 4. Venn diagram of BTG1-associated genes and DLBCL-associated genes. (A) Downregulated and (B) upregulated genes. BTG1, B-cell translocation gene 1; DLBCL, diffuse large B-cell lymphoma; DEG, differentially-expressed gene.

datasets (Fig. 2). Of the 407 BTG1-associated genes, 347 were upregulated and 60 were downregulated.

$B T G 1$ serves a role in $D L B C L$ progression. The association between BTG1 expression and DLBCL was then analyzed. Using Oncomine analysis, the expression of BTG1 was identified to be downregulated in DLBCL (31) (Fig. 3). Using the GSE65720 dataset and GEO2R analysis, a total of 22,187 DEGs were identified in DLBCL compared with normal lymphocytes. Overlapping analysis of the 407 BTG1-associated genes and the 22,187 DEGs revealed that 401 genes were BTG1-associated DLBCL genes (Fig. 4). Subsequently, GO and KEGG pathway analysis was performed to classify the 401 overlapping genes. The most significantly enriched GO terms were 'transcription' (GO: Biological process), 'nucleus' (GO: Cellular component) and 'protein binding' (GO: Molecular function) (Fig. 5). Additionally, KEGG pathway analysis revealed that the BTG1-associated DLBCL genes were involved in seven pathways, including 'Ribosome', 'Cell cycle' and 'B cell receptor signaling pathway' (Fig. 6). In summary, BTG1 may be involved in DLBCL progression.

Establishment of a PPI network and identification of hub genes. The STRING database and Cytoscape analysis were used to predict a potential interaction network for the BTG1-associated DLBCL genes. The PPI network was composed of 235 nodes and 601 edges, including 343 upregulated genes and 58 downregulated genes (Fig. 7). Additionally, when a degree $\geq 17$ was set as the cut-off point, 24 genes in the PPI network were identified as hub genes, including ubiquitin carboxyl extension protein 52 (UBA52), ribosomal protein (RP) L11, mitogen-activated protein kinase 1 (MAPK1) and exosome component 4.

Furthermore, 11 clusters were selected from a PPI network using MCODE, which revealed that the most significant cluster consisted of 21 nodes and 203 edges. Additionally, MCODE analysis demonstrated that each cluster contained one 'seed' gene (32), including RPL31, hect domain and RLD 4 and heterogeneous nuclear ribonucleoprotein A3 (Fig. 8).

\section{Discussion}

DLBCL is the most common lymphoid malignancy, with the incidence rate of lymphoma in China reported as 643/100,000 in 2012, and is part of a heterogeneous group of fast growing neoplasms, which exhibit an aggressive clinical course (33). Multi-agent chemotherapy has the potential to cure $\sim 40 \%$ of patients and combination with an anti-CD20 monoclonal antibody has further improved the treatment response for an additional $10-25 \%$ of patients (34). Despite improvements in therapy for DLBCL, $30 \%$ of patients do not respond to treatment attempts (35). The variation in prognosis for patients with DLBCL supports investigations of prognostic factors that can predict treatment response and the clinical course.

The BTG family serves a role in cancer, as BTG proteins can regulate the cell cycle (36). As a member of the BTG family, BTG1 has been identified to possess a t (q24;q22) translocation in B-cell chronic lymphocytic leukemia and serve as a biomarker for complete remission of acute lymphoblastic leukemia (37). Additionally, BTG1 is considered to be a tumor suppressor gene that is typically downregulated in various types of cancer, including colorectal, ovarian and renal cancer $(13,20,38)$. However, to the best of our knowledge, the role of BTG1 in DLBCL remains unclear. The present study performed systemic bioinformatics analysis to investigate the mechanism and gene network of BTG1 in DLBCL.

The present study investigated the association between BTG1 and clinical characteristics, as well as the diagnostic value of BTG1 for DLBCL. According to 470 samples obtained from the GSE31312 dataset, the expression level of BTG1 was associated with treatment response and IPI score. Furthermore, univariate and multivariate Cox regression analysis indicated that BTG1 expression level was a prognostic factor for overall survival and progression-free survival times. Although clinical data is missing for 28 patients, which may have certain effects on the results, it can be indicated that BTG1 is a protective factor in DLBCL.

A total of 401 BTG1-associated DLBCL genes were identified from the GSE31312, GSE10846 and GSE87371 datasets, consisting of 343 upregulated genes and 58 downregulated genes. These genes were enriched in seven pathways, including 'Ribosome', 'Cell cycle' and 'B cell receptor signaling pathway'. According to their degree in the PPI network, 24 genes were recognized as hub genes. The hub genes were associated with 'Ribosome' (RPL11, RPL5, RPS15, RPS14, RPL22 and RPL37), 'Cell cycle' (UBA52, ATM and Ras homolog family member H), 'MAPK pathway' (MAPK1), 'histone modification' (ASH1-like protein) and 'transcription/translation' (eukaryotic translation 
A

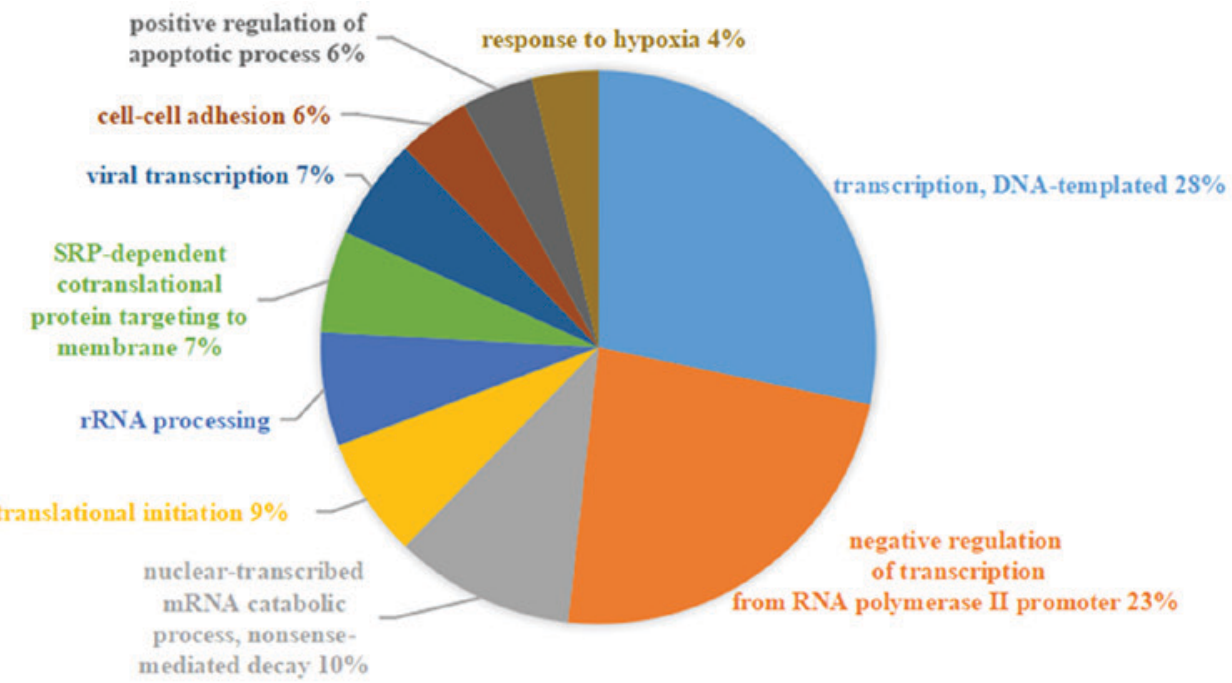

B
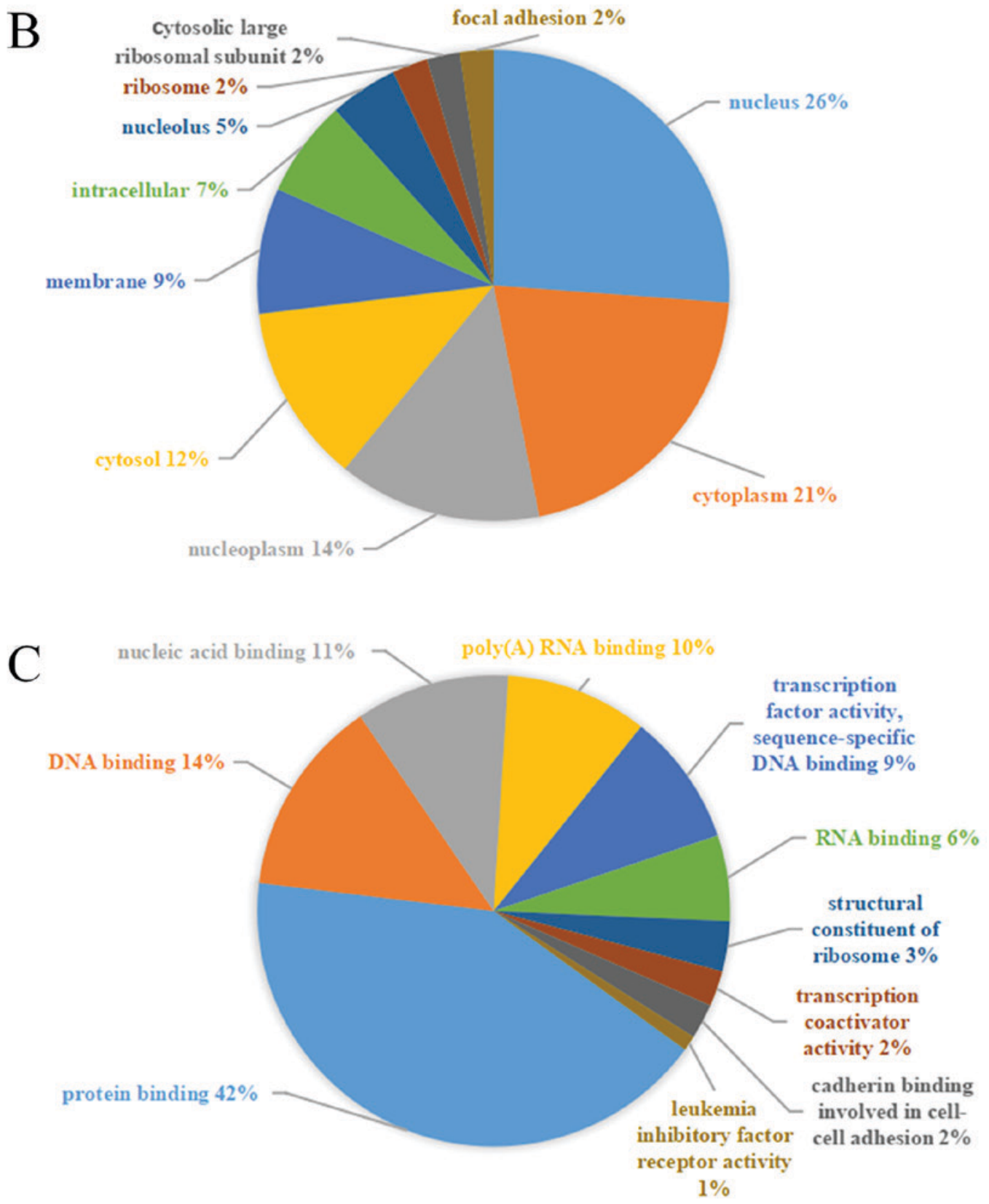

Figure 5. Enriched Gene Ontology terms for B-cell translocation gene 1-associated diffuse large B-cell lymphoma genes. (A) Biological process terms. (B) Cellular component terms. (C) Molecular function terms. 


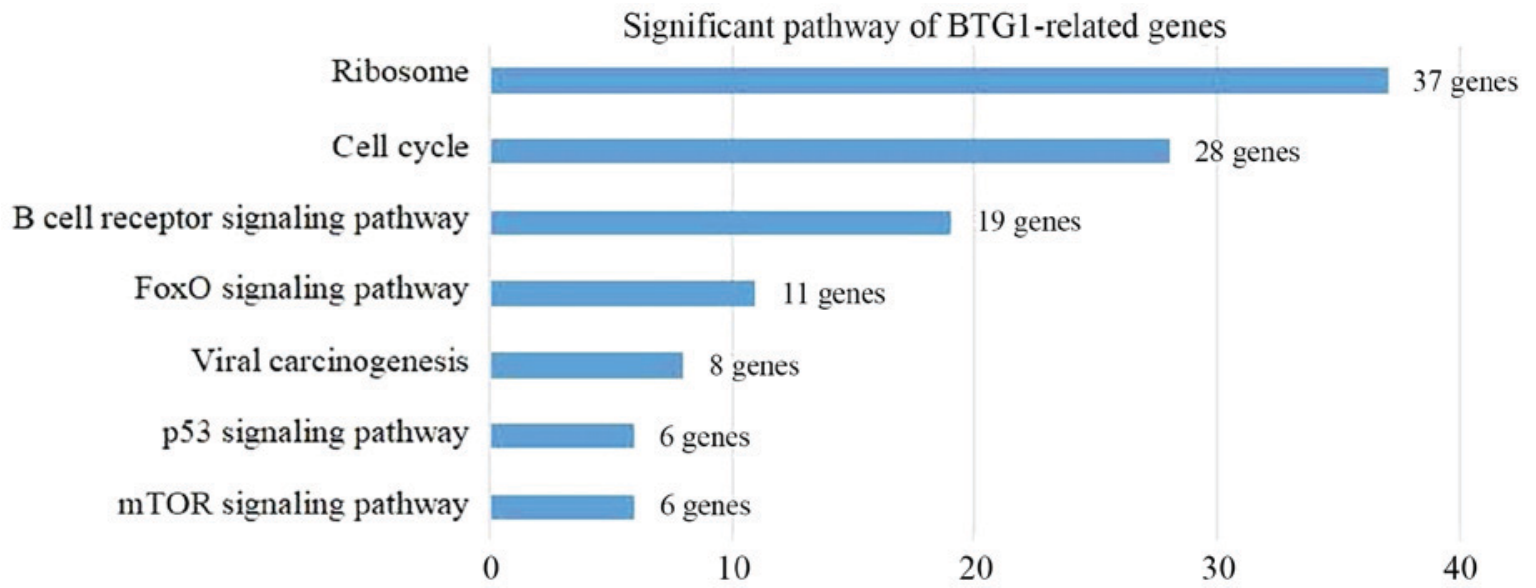

Figure 6. Enriched pathways for BTG1-associated diffuse large B-cell lymphoma genes. BTG1, B-cell translocation gene 1; FoxO, forkhead box O; mTOR, mammalian target of rapamycin.

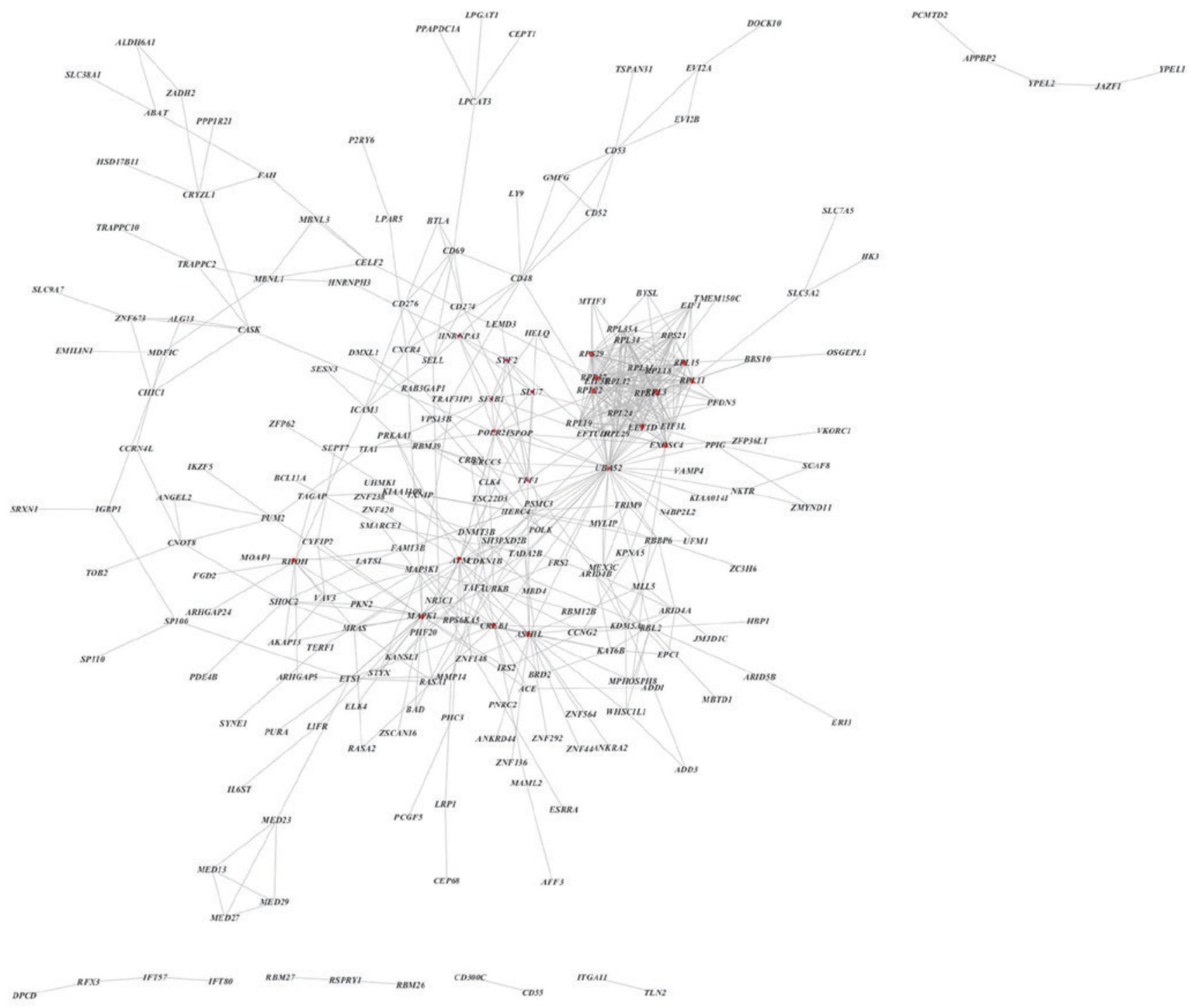

Figure 7. Protein-protein interaction network of B-cell translocation gene 1-associated genes in diffuse large B-cell lymphoma. Red nodes indicate hub genes.

initiation factor 3 subunit E, eukaryotic translation elongation factor $1 \delta$, transcription termination factor 1 , cAMP responsive element binding protein 1 and RNA polymerase II subunit F).
Notably, a panel of genes that encode RPs, including RPL11, RPL3, RPS29, RPL19, RPL15 and RPL12, were identified to be highly associated with the expression level of BTG1. Cancer 

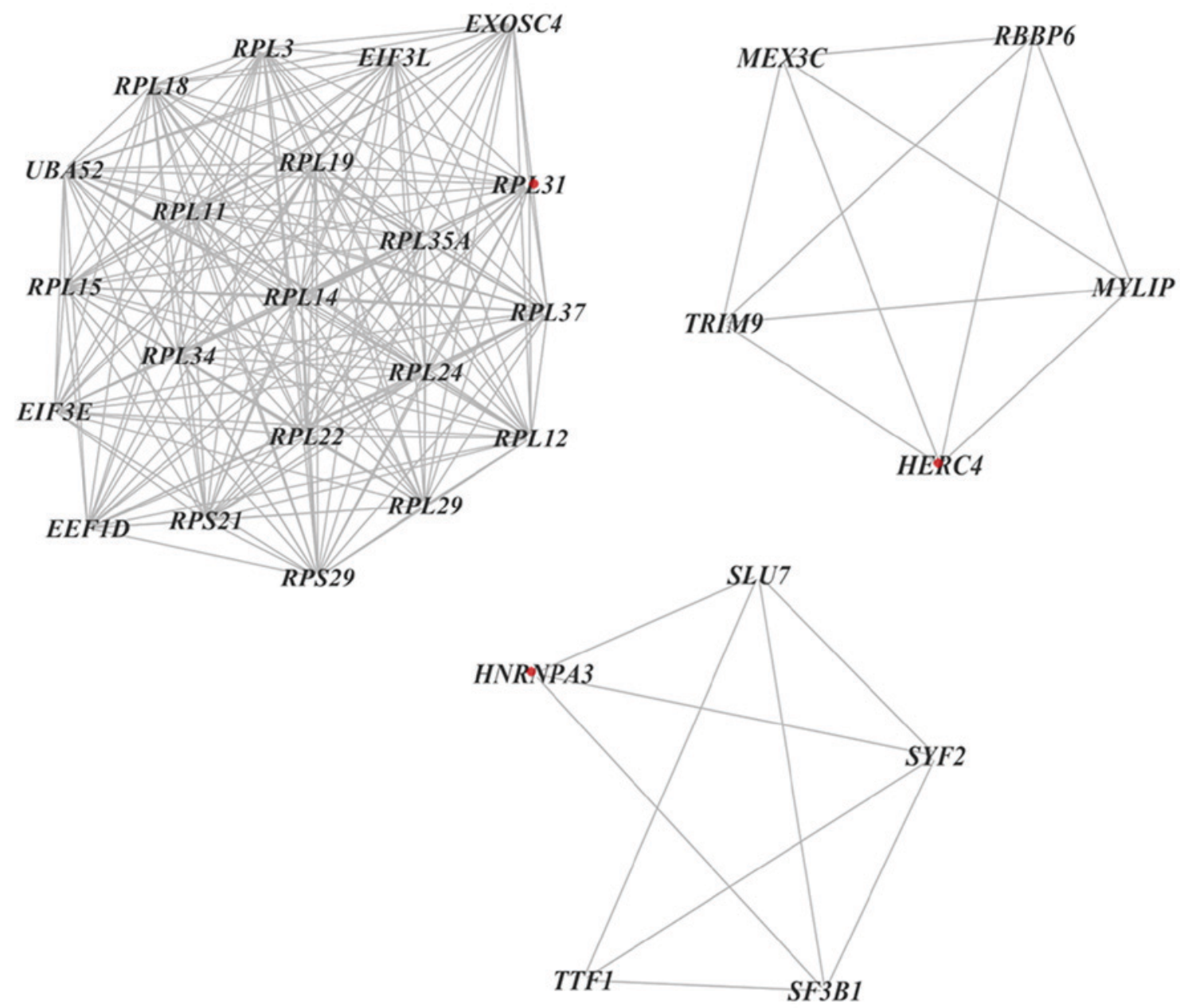

Figure 8. Three most significant clusters selected from the protein-protein interaction network. Red nodes indicate seed genes.

cells require large amounts of protein and increased protein synthesis, and consequently require efficient ribosome translational machinery (39). Therefore, a number of carcinogens and tumor suppressors, including p53, p21 and mMRPS36, frequently affect the growth of cancer cells by regulating ribosome biogenesis and protein synthesis (40). Numerous RPs, including RPL11, RPL5, RPL37, RPS15 and RPS14, have been identified to suppress tumor cell proliferation by regulating the mouse double minute (MDM) 2 homolog/MDMX-p53 cascade (41-44). RPL11 has also been revealed to suppress c-Myc activity and promote microRNA (miR)-24/miR-induced silencing complex-mediated c-Myc mRNA degradation (45). Additionally, mutations in certain RP-encoding genes, including RPL5 and RPL22, in tumors further indicates that RPs can be regarded as tumor suppressors (46).

A high degree of interaction was also observed between the hub genes UBA52, MAPK1 and BTG1. Ubiquitination is an important post-translational modification. UBA52 encodes a fusion protein, which consists of ubiquitin at the N-terminus and RPL40 at the C-terminus. UBA52 deficient cells exhibit inhibited protein synthesis and cell cycle arrest (47). As an ubiquitin-coding gene, UBA52 also serves a role in the regulation of the ribosomal protein complex (48). The MAPK signaling cascade is a pathway that mediates the proliferation and differentiation of hematopoietic cells. Among the MAPKs, MAPK1 serves a role in various mitogenic signaling pathways and participates in a diversity of cellular programs, including cell cycle progression and differentiation (49).

The majority of hub genes associated with BTG1 were identified to be involved in the ribosomal, cell cycle and p53 pathways. These results were consistent with GO and KEGG analysis of the BTG1-associated DLBCL genes. Other pathways identified by KEGG analysis included the BCR signaling pathway, the forkhead box $\mathrm{O}$ (FoxO) signaling pathway and the mTOR signaling pathway. DLBCL activates BCR signaling to maintain malignant growth and survival, which is mediated by NF- $\mathrm{NB}$ and other signals (12). The FoxO proteins are a subfamily of the fork head transcription factor family, which exhibit important roles in cell fate and tumor suppression (50). mTOR has been investigated for a number of years as a central regulator of cell growth, proliferation, survival and differentiation (51). The mechanism of BTG1 in DLBCL may involve these aforementioned pathways. However, potential mechanisms have not been investigated in DLBCL in vivo or in vitro. Therefore, further studies are required to support the results of the present study.

In conclusion, the present study indicated that BTG1 may be an independent prognostic factor for DLBCL and may serve a role in the progression and development of the disease. The aim of the present study was to predict the mechanism of BTG1 in DLBCL using bioinformatics analysis. It was identified that BTG1 may interact with RPs, UBA52, MAPK1 
and other genes to participate in the development of DLBCL, which would involve numerous tumor-associated signaling pathways. Future studies are required to verify the potential regulatory network proposed in the present study.

\section{Acknowledgements}

Not applicable.

\section{Funding}

No funding was received.

\section{Availability of data and material}

The datasets analyzed during the current study are available from the GEO database (http://www.ncbi.nlm.nih.gov/geo). All clinicopathological data analyzed during this study are included in the published GEO dataset, GSE31312 (25).

\section{Authors' contributions}

WYan and WYang designed the study and conducted bioinformatics analysis. WYan, SXL and HG performed statistical analysis, participated in data collection and drafted the manuscript. WYang supervised the scientific work and revising it critically amended the manuscript. She also gave final approval of the version to be published. All authors read and approved the final manuscript.

\section{Ethics approval and consent to participate}

Not applicable.

\section{Patient consent for publication}

Not applicable.

\section{Competing interests}

The authors declare that they have no competing interests.

\section{References}

1. Garciaz S, Coso D, Brice P and Bouabdallah R: Hodgkin and non-Hodgkin lymphoma of adolescents and young adults. Bull Cancer 103: 1035-1049, 2016 (In French).

2. Vega GG, Avilés-Salas A, Chalapud JR, Martinez-Paniagua M, Pelayo R, Mayani H, Hernandez-Pando R, Martinez-Maza O, Huerta-Yepez S, Bonavida B and Vega MI: P38 MAPK expression and activation predicts failure of response to $\mathrm{CHOP}$ in patients with diffuse large B-cell lymphoma. BMC Cancer 15: $722,2015$.

3. Zhang J, Wei B, Hu H, Liu F, Tu Y, Zhao M and Wu D: Preliminary study on decreasing the expression of FOXP3 with miR-155 to inhibit diffuse large B-cell lymphoma. Oncol Lett 14: 1711-1718, 2017.

4. Hu X, Zeng M, Yang SE, Liang X, Ding SS, Guo L, Li S and Wen SJ: Efficacy of rituximab combined with CHOP for treating patients with diffuse large B-cell lymphoma. Medicine (Baltimore) 96: e8494, 2017.

5. De Paepe P, Achten R, Verhoef G, Wlodarska I, Stul M, Vanhentenrijk V, Praet M and De Wolf-Peeters C: Large cleaved and immunoblastic lymphoma may represent two distinct clinicopathologic entities within the group of diffuse large B-cell lymphomas. J Clin Oncol 23: 7060-7068, 2005.
6. Tumwine LK, Agostinelli C, Campidelli C, Othieno E, Wabinga H, Righi S, Falini B, Piccaluga PP, Byarugaba W and Pileri SA: Immnohistochemical and other prognostic factors in B cell non Hodgkin lymphoma patients, Kampala, Uganda. BMC Clin Pathol 9: 11, 2009.

7. Offner F, Samoilova O, Osmanov E, Eom HS, Topp MS, Raposo J, Pavlov V, Ricci D, Chaturvedi S, Zhu E, et al: Frontline rituximab, cyclophosphamide, doxorubicin, and prednisone with bortezomib (VR-CAP) or vincristine (R-CHOP) for non-GCB DLBCL. Blood 126: 1893-1901, 2015.

8. Leonard JP, Kolibaba KS, Reeves JA, Tulpule A, Flinn IW, Kolevska T, Robles R, Flowers CR, Collons R, DiBella NJ, et al: Randomized phase II study of R-CHOP with or without bortezomib in previously untreated patients with non-germinal center B-cell-like diffuse large B-cell lymphoma. J Clin Oncol 35: 3538-3546, 2017.

9. Murthy SL, Hitchcock MA, Endicott-Yazdani TR, Watson JT and Krause JR: Epstein-barr virus-positive diffuse large B-cell lymphoma. Proc (Bayl Univ Med Cent) 30: 443-444, 2017.

10. van Krieken JH: New developments in the pathology of malignant lymphoma: A review of the literature published from May to August 2017. J Hematop 10: 65-73, 2017.

11. Bognar MK, Vincendeau M, Erdmann T, Seeholzer T, Grau M, Linnemann JR, Ruland J, Scheel CH, Lenz P, Ott G, et al: Oncogenic CARMA1 couples NF- $\kappa \mathrm{B}$ and $\beta$-catenin signaling in diffuse large B-cell lymphomas. Oncogene 35: 4269-4281, 2016.

12. Young RM, Shaffer AL III, Phelan JD and Staudt LM: B cell receptor signaling in diffuse large B cell lymphoma. Semin Hematol 52: 77-85, 2015.

13. Zhao Y, Gou WF, Chen S, Takano Y, Xiu YL and Zheng HC: BTG1 expression correlates with the pathogenesis and progression of ovarian carcinomas. Int J Mol Sci 14: 19670-19680, 2013.

14. Rouault JP, Rimokh R, Tessa C, Paranhos G, Ffrench M, Duret L, Garoccio M, Germain D, Samarut J and Magaud JP: BTG1, a member of a new family of antiproliferative genes. EMBO J 11: 1663-1670, 1992.

15. Mauxion F, Chen CY, Séraphin B and Shyu AB: BTG/TOB factors impact deadenylases. Trends Biochem Sci 34: 640-647, 2009.

16. Busson M, Carazo A, Seyer P, Grandemange S, Casas F, Pessemesse L, Rouault JP, Wrutniak-Cabello C and Cabello G: Coactivation of nuclear receptors and myogenic factors induces the major BTG1 influence on muscle differentiation. Oncogene 24: 1698-1710, 2005.

17. Yin P, Song G and Jiang Z: Cisplatin suppresses proliferation, migration and invasion of nasopharyngeal carcinoma cells in vitro by repressing the Wnt/ $\beta$-catenin/Endothelin- 1 axis via activating $B$ cell translocation gene 1 . Cancer Chemother Pharmacol 81: 863-872, 2018.

18. Rouaul JP, Prévôt D, Berthet C, Birot AM, Billaud M, Magaud JP and Corbo L: Interaction of BTG1 and p53-regulated BTG2 gene products with mCaf1, the murine homolog of a component of the yeast CCR4 transcriptional regulatory complex. J Biol Chem 273: 22563-22569, 1998.

19. Tijchon E, van Emst L, Yuniati L, van lngen Schenau D, Gerritsen M, van der Meer LT, Williams O, Hoogerbrugge PM, Scheijen B and van Leeuwen FN: Tumor suppressor BTG1 limits activation of BCL6 expression downstream of ETV6-RUNX1. Exp Hematol 60: 57-62.e3, 2018.

20. Liu C, Tao T, Xu B, Lu K, Zhang L, Jiang L, Chen S, Liu D, Zhang X, Cao N and Chen M: BTG1 potentiates apoptosis and suppresses proliferation in renal cell carcinoma by interacting with PRMT1. Oncol Lett 10: 619-624, 2015.

21. He C, Yu T, Shi Y, Ma C, Yang W, Fang L, Sun M, Wu W, Xiao F, Guo F, et al: MicroRNA 301A promotes intestinal inflammation and colitis-associated cancer development by inhibiting BTG1. Gastroenterology 152: 1434-1448.e15, 2017.

22. Corjay MH, Kearney MA, Mnzer DA, Diamond SM and Stoltenborg JK: Antiproliferative gene BTG1 is highly expressed in apoptotic cells in macrophage-rich areas of advanced lesions in Watanabe heritable hyperlipidemic rabbit and human. Lab Invest 78: 847-858, 1998.

23. Scheijen B, Boer JM, Marke R, Tijchon E, van lngen Schenau D, Waanders E, van Emst L, van der Meer LT, Pieters R, Escherich G, et al: Tumor suppressors BTG1 and IKZF1 cooperate during mouse leukemia development and increase relapse risk in B-cell precursor acute lymphoblastic leukemia patients. Haematologica 102: 541-551, 2017.

24. Tijchon E, van Emst L, Yuniati L, van lngen Schenau D, Havinga J, Rouault JP, Hoogerbrugge PM, van Leeuwen FN and Scheijen B: Tumor suppressors BTG1 and BTG2 regulate early mouse B-cell development. Haematologica 101: e272-e276, 2016. 
25. Visco C, Li Y, Xu-Monette ZY, Miranda RN, Green TM, Li Y, Tzankov A, Wen W, Liu WM, Kahl BS, et al: Comprehensive gene expression profiling and immunohistochemical studies support application of immunophenotypic algorithm for molecular subtype classification in diffuse large B-cell lymphoma: A report from the International DLBCL Rituximab-CHOP consortium program study. Leukemia 26: 2103-2113, 2012.

26. Simon R, Durrleman S, Hoppe RT, Bonadonna G, Bloomfield CD, Rudders RA, Cheaon BD and Berard CW: The non-hodgkin lymphoma pthologic classification profect. Long-term follow-up of 1153 patients with non-Hodgkin lymphomas. Ann Intern Med 109: 939-945, 1988.

27. Meyer PN, Fu K, Greiner TC, Smith LM, Delabie J, Gascoyne RD, Ott G, Rosenwald A, Braziel RM, Campo E, et al: Immunohistochemical methods for predicting cell of origin and survival in patients with diffuse large B-cell lymphoma treated with rituximab. J Clin Oncol 29: 200-207, 2011.

28. Dubois S and Jardin F: Novel molecular classifications of DLBCL. Nat Rev Clin Oncol 15: 474-476, 2018

29. Liao YX, Zhang ZP, Zhao J and Liu JP: Effects of fibronectin 1 on cell proliferation, senescence and apoptosis of human glioma cells through the PI3K/AKT signaling pathway. Cell Physiol Biochem 48: 1382-1396, 2018.

30. Zang Y, Gu L, Zhang Y, Wang Y and Xue F: Identification of key genes and pathways in uterine leiomyosarcoma through bioinformatics analysis. Oncol Lett 15: 9361-9368, 2018.

31. Rosenwald A, Wright G, Chan WC, Connors JM, Campo E, Fisher RI, Gascoyne RD, Muller-Hermelink HK, Smeland EB Giltnane JM, et al: The use of molecular profiling to predict survival after chemotherapy for diffuse large-B-cell lymphoma. N Engl J Med 346: 1937-1947, 2002.

32. Pan Y, Lu L, Chen J, Zhong Y and Dai Z: Identification of potential crucial genes and construction of microRNA-mRNA negative regulatory network in osteosarcoma. Hereditas 155: 21, 2018.

33. Chen $\mathrm{W}$,Zheng R, Zeng $\mathrm{H}$ and Zhang $\mathrm{S}$ : The incidence and mortality of major cancers in China, 2012. Chin J Cancer 35: 73, 2016.

34. Wu J, Song Y, Su L, Xu L, Chen T, Zhao Z, Zhang M, Li W, Hu Y, Zhang X, et al: Rituximab plus chemotherapy as first-line treatment in Chinese patients with diffuse large B-cell lymphoma in routine practice: A prospective, multicentre, non-interventional study. BMC Cancer 16: 537, 2016.

35. Yamauchi T, Tasaki T, Tai K, Ikegaya S, Takagi K, Negoro E, Kishi S, Yoshida A, Iwasaki H and Ueda T: Prognostic effect of peripheral blood cell counts in advanced diffuse large B-cell lymphoma treated with R-CHOP-like chemotherapy: A single institution analysis. Oncol Lett 9: 851-856, 2015.

36. Huang Y, Zheng J, Tan T, Song L, Huang S, Zhang Y, Lin L, Liu J, Zheng P, Chen X, et al: BTG1 low expression in pancreatic ductal adenocarcinoma is associated with a poorer prognosis. Int J Biol Markers 33: 189-194, 2018.

37. Rimokh R, Rouault JP, Wahbi K, Gadoux M, Lafage M, Archimbaud E, Charrin C, Gentilhomme O, Germain D, Samarut $\mathrm{J}$, et al: A chromosome 12 coding region is juxtaposed to the MYC protooncogene locus in a $\mathrm{t}(8 ; 12)(\mathrm{q} 24 ; \mathrm{q} 22)$ translocation in a case of B-cell chronic lymphocytic leukemia. Genes Chromosomes Cancer 3: 24-36, 1991
38. Zhao S, Chen SR, Yang XF, Shen DF, Takano Y, Su RJ and Zheng HC: BTG1 might be employed as a biomarker for carcinogenesis and a target for gene therapy in colorectal cancers. Oncotarget 8: 7502-7520, 2017.

39. Zhou X, Liao WJ, Liao JM, Liao P and Lu H: Ribosomal proteins: Functions beyond the ribosome. J Mol Cell Biol 7: 92-104, 2015

40. Chen YC, Chang MY, Shiau AL, Yo YT and Wu CL: Mitochondrial ribosomal protein S36 delays cell cycle progression in association with $\mathrm{p} 53$ modification and p21(WAF1/CIP1) expression. J Cell Biochem 100: 981-190, 2007.

41. Lohrum MA, Ludwig RL, Kubbutat MH, Hanlon M and Vousden KH: Regulation of HDM2 activity by the ribosomal protein L11. Cancer Cell 3: 577-587, 2003.

42. Dai MS and Lu H: Inhibition of MDM2-mediated p53 ubiquitination and degradation by ribosomal protein L5. J Biol Chem 279: 44475-44482, 2004

43. Daftular L, Zhu Y, Jacq X and Prives C: Ribosomal proteins RPL37, RPS15 and RPS20 regulate the Mdm2-p53-MdmX network. PLoS One 8: e68667, 2013.

44. Zhou X, Hao Q, Liao J, Zhang Q and Lu H: Ribosomal protein S14 unties the MDM2-p53 loop upon ribosomal stress. Oncogene 32: 388-396, 2013.

45. Challagundla KB, Sun XX, Zhang X, DeVine T, Zhang Q, Sears RC and Dai MS: Ribosomal protein L11 recruits $\mathrm{miR}-24 / \mathrm{miRISC}$ to repress c-Myc expression in response to ribosomal stress. Mol Cell Biol 31: 4007-4021, 2011.

46. De Keesmaecker K, Atak ZK, Li N, Vicente C, Patchett S, Girardi T, Gianfelici V, Geerdens E, Clappier E, Porcu M, et al: Exome sequencing identifies mutation in CNOT3 and ribosomal genes RPL5 and RPL10 in T-cell acute lymphoblastic leukemia. Nat Genet 45: 186-190, 2013.

47. Mao J, O'Gorman C, Sutovsky M, Zigo M, Wells KD and Sutovsky P: Ubiquitin A-52 residue ribosomal protein fusion product 1 (Uba52) is essential for preimplantation embryo development. Biol Open 7: pii: bio035717, 2018.

48. Kobayashi M, Oshima S, Maeyashiki C, Nibe Y, Otsubo K, Matsuzawa $Y$, Nemoto $Y$, Nagaishi T, Okamoto R, Tsuchiya $\mathrm{K}$, et al: The ubiquitin hybrid gene UBA52 regulates ubiquitination of ribosome and sustains embryonic development. Sci Rep 6: 36780, 2016.

49. Gregorj C, Ricciardi MR, Petrucci MT, Scerpa MC, De Cave F, Fazi P, Vignetti M, Vitale A, Mancini M,Cimino G, et al: ERK1/2 phosphorylation is an independent predictor of complete remission in newly diagnosed adult acute lymphoblastic leukemia. Blood 109: 5473-5476, 2007.

50. Farhan M, Wang H, Gaur U, Little PJ, Xu J and Zheng W: FOXO signaling pathways as therapeutic targets in cancer. Int J Biol Sci 13: 815-827, 2017

51. Zhou H and Huang S: mTOR signaling in cancer cell motility and tumor metastasis. Crit Rev Eukaryot Gene Expr 20: 1-16, 2010.

c) (7) $\Theta$ This work is licensed under a Creative Commons Attribution-NonCommercial-NoDerivatives 4.0 International (CC BY-NC-ND 4.0) License. 